\section{REFERENCES AND NOTES}

1. A. Kilian et al., Hum. Mol. Genet. 6, 2011-2019 (1997).

2. C. Günes, S. Lichtsteiner, A. P. Vasserot, C. Englert, Cancer Res. 60, 2116-2121 (2000).

3. J. W. Shay, S. Bacchetti, Eur. J. Cancer 33, 787-791 (1997).

4. S. Horn et al., Science 339, 959-961 (2013).

5. F. W. Huang et al., Science 339, 957-959 (2013).

6. P. J. Killela et al., Proc. Natl. Acad. Sci. U.S.A. 110, 6021-6026 (2013).

7. N. Weinhold, A. Jacobsen, N. Schultz, C. Sander, W. Lee, Nat. Genet. 46, 1160-1165 (2014).

8. P. S. Rachakonda et al., Proc. Natl. Acad. Sci. U.S.A. 110, 17426-17431 (2013)

9. B. Heidenreich et al., Nat. Commun. 5, 3401 (2014).

10. J. C. Nault et al., Nat. Commun. 4, 2218 (2013).

11. A. Tallet et al., Oncogene 33, 3748-3752 (2014).

12. J. Vinagre et al., Nat. Commun. 4, 2185 (2013).

13. Y. Allory et al., Eur. Urol. 65, 360-366 (2014).

14. J.-I. Park et al., Nature 460, 66-72 (2009).

15. I. Listerman, F. S. Gazzaniga, E. H. Blackburn, Mol. Cell. Biol. 34, 280-289 (2014)

16. C. D. Hurst, F. M. Platt, M. A. Knowles, Eur. Urol. 65, 367-369 (2014).

17. Y. Lin et al., Clin. Cancer Res. 2, 929-932 (1996)

18. Y. Cao, T. M. Bryan, R. R. Reddel, Cancer Sci. 99, 1092-1099 (2008).

19. K. A. Hoadley et al., Cell 158, 929-944 (2014).

20. A. Adey et al., Nature 500, 207-211 (2013).

21. E. Kjeldsen, D. Tordrup, G. M. Hübner, B. R. Knudsen, F. F. Andersen, Anticancer Res. 30, 3257-3265 (2010).

22. M. Meyerson et al., Cell 90, 785-795 (1997).

23. L. H. Li, C. Nerlov, G. Prendergast, D. MacGregor, E. B. Ziff, EMBO J. 13, 4070-4079 (1994)

24. Y. Gui et al., Nat. Genet. $43,875-878$ (2011).

25. L. Xi, T. R. Cech, Nucleic Acids Res. 42, 8565-8577 (2014).

26. S. B. Cohen, R. R. Reddel, Nat. Methods 5, 355-360 (2008).

27. H. B. Grossman et al., N. Engl. J. Med. 349, 859-866 (2003).

28. W. J. Kim et al., Mol. Cancer 9, 3 (2010).

29. M. Sanchez-Carbayo, N. D. Socci, J. Lozano, F. Saint, C. Cordon-Cardo, J. Clin. Oncol. 24, 778-789 (2006).

30. J. J. Gildea, W. L. Golden, M. A. Harding, D. Theodorescu, Genes Chromosomes Cancer 27, 252-263 (2000).

31. J. J. Gildea et al., Cancer Res. 62, 6418-6423 (2002).

32. C. M. Heaphy et al., Am. J. Pathol. 179, 1608-1615 (2011)

33. The use of microarray data sets to identify alternative mechanisms of TERT reactivation employed by those cell lines that were WT at positions -124 and -146 was unsuccessful. Average mRNA levels were roughly similar for (i) the ETS1 and ETS2 transcription factors ( 1.1-fold higher in the WT group), (ii) the c-MYC transcription factor ( 1.4-fold higher in the WT group), and (iii) the core components of the Polycomb repressive complex 2 (between $\sim 0.91$-fold and $\sim 1.3$-fold higher in the WT group).

34. J. Nandakumar et al., Nature 492, 285-289 (2012).

\section{ACKNOWLEDGMENTS}

We thank C. Owens for providing the UC23, as well as detailed information on their origins and growth conditions. This work was supported by NIH grants CA075115 and CA104106 to D.T R01 GM099705 to T.R.C., and T32 GM08759 to L.X. S.B.C. is supported by Cancer Council NSW (Australia). T.R.C. is an investigator of the Howard Hughes Medical Institute and is on the board of directors of Merck.

\section{SUPPLEMENTARY MATERIALS}

www.sciencemag.org/content/347/6225/1006/suppl/DC1 Materials and Methods

Supplementary Text

Figs. S1 to S7

Tables S1 to S3

References $(35,36)$

20 August 2014; accepted 24 January 2015

Published online 5 February 2015:

10.1126/science. 1260200

\title{
Transcribed enhancers lead waves of coordinated transcription in transitioning mammalian cells
}

Erik Arner, ${ }^{*} \dagger$ Carsten O. Daub, ${ }^{*}+$ Kristoffer Vitting-Seerup,${ }^{*} \dagger$ Robin Andersson, ${ }^{*} \dagger$ Berit Lilje, Finn Drabløs, Andreas Lennartsson, Michelle Rönnerblad, Olga Hrydziuszko, Morana Vitezic, Tom C. Freeman, Ahmad M. N. Alhendi, Peter Arner, Richard Axton, J. Kenneth Baillie, Anthony Beckhouse, Beatrice Bodega, James Briggs, Frank Brombacher, Margaret Davis, Michael Detmar, Anna Ehrlund, Mitsuhiro Endoh, Afsaneh Eslami, Michela Fagiolini, Lynsey Fairbairn, Geoffrey J. Faulkner, Carmelo Ferrai, Malcolm E. Fisher, Lesley Forrester, Daniel Goldowitz, Reto Guler, Thomas Ha, Mitsuko Hara, Meenhard Herlyn, Tomokatsu Ikawa, Chieko Kai, Hiroshi Kawamoto, Levon M. Khachigian, S. Peter Klinken, Soichi Kojima, Haruhiko Koseki, Sarah Klein, Niklas Mejhert, Ken Miyaguchi, Yosuke Mizuno, Mitsuru Morimoto, Kelly J. Morris, Christine Mummery, Yutaka Nakachi, Soichi Ogishima, Mariko Okada-Hatakeyama, Yasushi Okazaki, Valerio Orlando, Dmitry Ovchinnikov, Robert Passier, Margaret Patrikakis, Ana Pombo, Xian-Yang Qin, Sugata Roy, Hiroki Sato, Suzana Savvi, Alka Saxena, Anita Schwegmann, Daisuke Sugiyama, Rolf Swoboda, Hiroshi Tanaka, Andru Tomoiu, Louise N. Winteringham, Ernst Wolvetang, Chiyo Yanagi-Mizuochi, Misako Yoneda, Susan Zabierowski, Peter Zhang, Imad Abugessaisa, Nicolas Bertin, Alexander D. Diehl, Shiro Fukuda, Masaaki Furuno, Jayson Harshbarger, Akira Hasegawa, Fumi Hori, Sachi Ishikawa-Kato, Yuri Ishizu, Masayoshi Itoh, Tsugumi Kawashima, Miki Kojima, Naoto Kondo, Marina Lizio, Terrence F. Meehan, Christopher J. Mungall, Mitsuyoshi Murata, Hiromi Nishiyori-Sueki, Serkan Sahin, Sayaka Nagao-Sato, Jessica Severin, Michiel J. L. de Hoon, Jun Kawai, Takeya Kasukawa, Timo Lassmann, Harukazu Suzuki, Hideya Kawaji, $\uparrow$ Kim M. Summers, $\uparrow$ Christine Wells, $\uparrow$ FANTOM Consortium, David A. Hume, $\uparrow \ddagger$ Alistair R. R. Forrest, $\uparrow \ddagger$ Albin Sandelin, $\uparrow \ddagger$ Piero Carninci, $\nmid \ddagger$ Yoshihide Hayashizaki $\nmid \ddagger$

Although it is generally accepted that cellular differentiation requires changes to transcriptional networks, dynamic regulation of promoters and enhancers at specific sets of genes has not been previously studied en masse. Exploiting the fact that active promoters and enhancers are transcribed, we simultaneously measured their activity in 19 human and 14 mouse time courses covering a wide range of cell types and biological stimuli. Enhancer RNAs, then messenger RNAs encoding transcription factors, dominated the earliest responses. Binding sites for key lineage transcription factors were simultaneously overrepresented in enhancers and promoters active in each cellular system. Our data support a highly generalizable model in which enhancer transcription is the earliest event in successive waves of transcriptional change during cellular differentiation or activation.

R egulated transcription initiation underlies state changes in cell phenotype and is coordinated by transcription factors binding to gene-proximal promoters or distal regulatory regions such as enhancers. The interaction between enhancers and transcription induction during cellular differentiation has been cited as one of the outstanding mysteries of modern biology (1). Enhancer chromatin landscapes change drastically between developing tissues and differentiated cells (2-4). Active en-

The list of author affiliations is available in the Supplementary Text. *These authors contributed equally to this work. FFANTOM5 Phase 2 Core authors. ‡Corresponding author. E-mail: david. hume@roslin.ed.ac.uk (D.A.H.); forrest@gsc.riken.jp (A.R.R.F.); albin@binf.ku.dk (A.S.); carninci@riken.jp (P.C.); yosihide@gsc. riken.jp (Y.H.) hancers initiate production of RNAs (eRNAs) (5) and enhancer action during differentiation can be assessed by sequencing of steady-state $(6,7)$ or nascent RNA (8-10), demonstrating that eRNA and target gene expression are correlated. eRNA production is also correlated to physical proximity between enhancers and promoters $(8,9)$. However, the general temporal relationship between enhancer and promoter activation across biological system is unknown.

Genome-scale $5^{\prime}$ rapid amplification of cDNA ends (cap analysis of gene expression, or CAGE) detects transcription start sites (TSSs), including the bidirectional TSS characteristic of active enhancers (11). Based on a large set of reporter assays, CAGE-defined enhancers are two to three times as likely to validate (12) as untranscribed chromatin-defined enhancer candidates from 
the ENCODE (Encyclopedia of DNA Elements) consortium (13). Here, we used CAGE to dissect the relationship between dynamic changes in mRNA and eRNA in 33 time courses of differentiation and activation. The time courses included stem cells (embryonic, induced pluripotent, trophoblastic, and mesenchymal stem cells) and committed progenitors undergoing terminal differentiation toward mesodermal, endodermal, and ectodermal fates, as well as fully differentiated primary cells and cell lines responding to stimuli (growth factors and pathogens) (Fig. 1, A and B; tables S1 to S3; and supplementary methods). In total, 1189 CAGE libraries from
408 distinct time points in the 33 time courses were analyzed (Fig. 1B and auxiliary data tables S1 and S2). Differentiation or response to stimulus was assessed by monitoring cell morphology changes, reproducible induction of known lineage markers, and similarity of the end-point transcriptome to differentiated cells from the steadystate samples of FANTOM5 (14) (auxiliary data table S1).

The current data expand the set of known human and mouse core promoters from the FANTOM5 body-wide steady-state atlas (14) to 201,802 and 158,966 , and the set of transcribed enhancers to 65,423 and 44,459 . Of all identi- fied core promoters in human and mouse, $51 \%$ and $61 \%$ varied significantly in expression in at least one time course. Out of the 103,355 differentially expressed human promoters, 80,152 were within genes on the same strand. Of these, 55,626 are potential alternative promoters (see supplementary methods), overlapping a total of 13,138 genes. We found 65 human genes that had a dynamic switch between alternative promoters within a time course, leading to exclusion of exons encoding protein domains (table S4).

Of all enhancers identified in FANTOM5, 42,274 human (65\%) and 34,338 mouse (77\%) enhancers were expressed in at least one CAGE library in
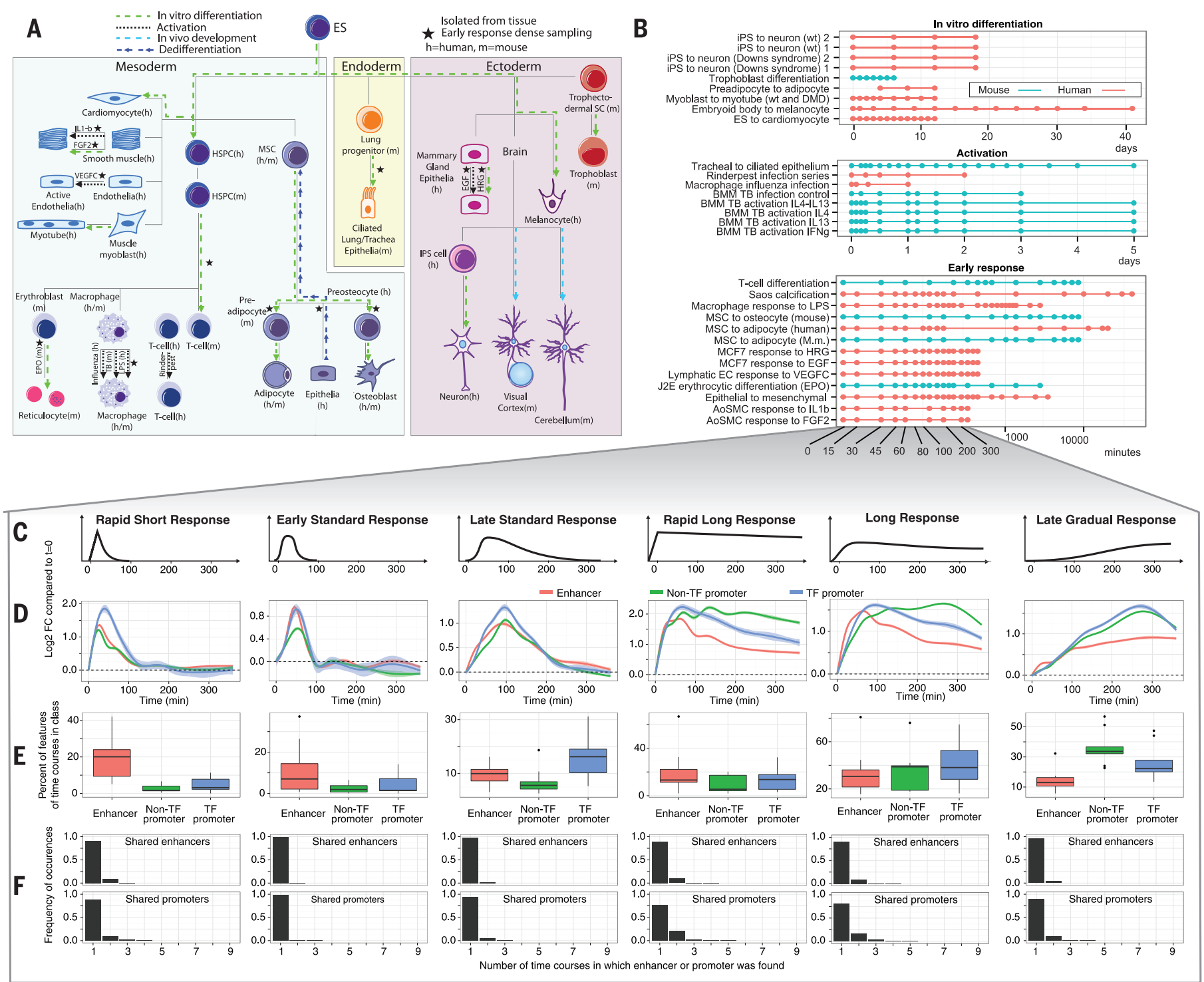

Fig. 1. Time course design and definition of response classes. (A) Schematic illustration of the time course experiments included in the study, arranged according to a development tree. Germ layers are shown as boxes. Black stars indicate time series sampled with high resolution. (B) Overview of time courses according to sampling strategy. The $x$ axis indicates time after induction. Each dot indicates CAGE sampling, typically done in biological triplicates. (C) Stylistic representation of each of the major up-regulated response patterns (classes) identified as described in the main text. The $y$ axis shows $\log _{2}$ fold change versus time 0; the $x$ axis shows time in minutes. (D) Mean expression $\log _{2}$ fold change across time courses for enhancers and promoters classified into each response pattern [as in (C)]. The 95\% confidence intervals of means are shown. (E) Boxplots of fractions showing the preference for enhancers, TF promoters, and other promoters for respective response class. (F) Overlap between time courses in terms of enhancers and promoters in respective class. Barplots show the frequency ( $y$ axis) of the number of time courses (out of 9) sharing a specific feature ( $x$ axis). 
the current study. Of these, 5371 (13\%) human and $6824(20 \%)$ mouse enhancers changed expression significantly over time in at least one time course. Most of these enhancer changes were time-course specific ( $56 \%$ in human, $67 \%$ in mouse). In contrast, the fraction of promoters regulated in only a single time course was smaller ( $29 \%$ in human, $33 \%$ in mouse).
We profiled 13 cellular systems with high temporal resolution within the first hours of cellular induction (Fig. 1B). We focused on the first 6 hours in nine of these time courses (five human and four mouse having sufficient numbers of dynamic promoters and enhancers; table S1).

Based on unsupervised clustering, we identified a set of distinct response pattern classes, shared
A

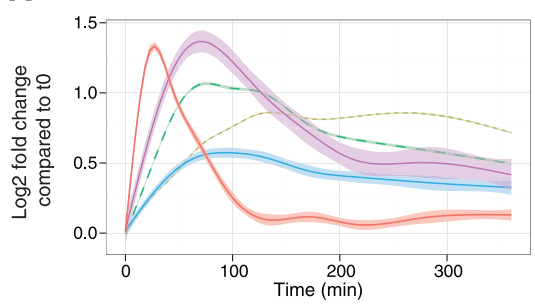

Rapid short response enhancers

Dynamic promoters within $200 \mathrm{~kb}$ of classified enhancers Dynamic TF promoters within $200 \mathrm{~kb}$ of classified enhancers Dynamic promoters within $200 \mathrm{~kb}$ of random enhancers

i= Dynamic TF promoters within $200 \mathrm{~kb}$ of random enhancers
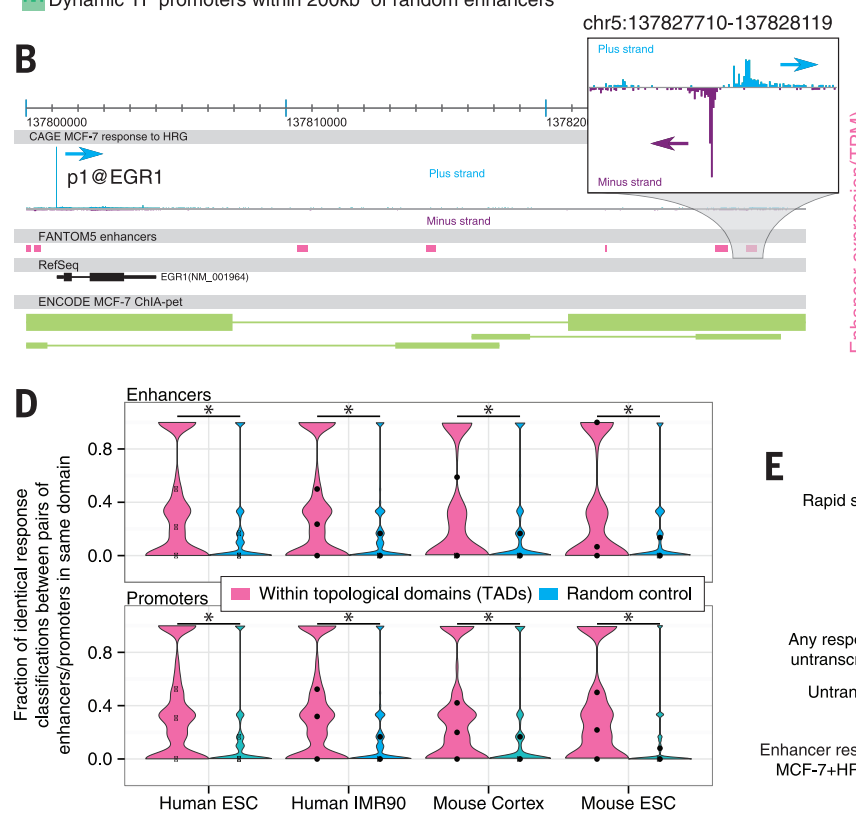

E

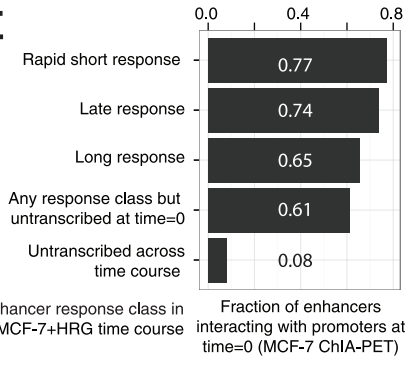

Fig. 2. Temporal shifts between enhancer and promoter activity. (A) Smoothed mean expression over time for all enhancers classified into the rapid short response group and all differentially expressed proximal $( \pm 200 \mathrm{~kb})$ promoters, split by gene type. Controls for class specificity (dotted lines) constitute promoters proximal to randomly sampled enhancers from other classes. Shaded areas indicate 95\% confidence intervals. (B) Example of expression timing in an enhancer-promoter pair (EGR1), showing activation of enhancers before promoter activation. MCF-7 ChIA-PET interaction data are visualized at the bottom as green lines; each line represents a cluster of ChIA-PET paired tags consisting of at least three pairs, where line end thickness is proportional to the number of paired tags in the cluster. Right panel shows the expression level of promoter and enhancer in MCF-7 cells after induction with HRG. Error bars indicate SD. (C) Left: Distribution of center of mass (CM) of expression changes (see main text) for enhancers, TF promoters, and promoters of other genes. Right: difference in CM ("shift") between enhancers-promoter pairs linked by proximity $( \pm 200 \mathrm{~kb})$ split by gene type. Black dots indicate $25 \mathrm{th}, 50 \mathrm{th}$, and 75 th percentiles. Asterisks indicate significance $\left(P<1.0 \times 10^{-106}\right.$, Mann-Whitney $U$ test). (D) The similarity of enhancer or promoter response classification (Fig. 1C) within each TAD was analyzed by calculating the frequency of identically classified enhancers or promoters in all pairwise comparisons. Frequency distributions are shown as violin plots. Controls are made by randomly sampling the same number of enhancers or promoters and calculating the classification similarity as above (repeated 100 times for each TAD). Asterisks indicate significance $(P<0.01$, Mann-Whitney $U$ test); dots represent percentiles, as in (C). (E) Fraction of enhancers that interact (by RNAPII-ChIA-PET) with promoters in unstimulated MCF-7 cells, split by enhancer response class in the MCF-7+HRG time course. by multiple time courses, by analyzing expression fold changes versus time 0 in each time course. For each response class, we defined specific expression rules (fig. S1), enabling consistent response class labeling of any dynamically transcribed enhancer or TSS in a time-course-specific fashion (figs. S2 to S4). Transcription factor (TF) promoters were analyzed as a distinct group. Because most enhancers and promoters that were dynamically changing in this set were up-regulated over time (fig. S5), we focused on the six up-regulated response classes (Fig. 1C).

Multiple enhancers, TF promoters, and nonTF promoters were found in all response classes (Fig. 1D, fig. S6, and auxiliary data table S3), but with different preferences. Enhancers were more common in the early peaking classes ("rapid short," "early standard," and "rapid long" responses). TF promoters were generally induced after enhancers (preferring the "late standard" response and "long response" classes) and non-TF promoters were most common in the "late gradual response" class that increased gradually with time (Fig. 1E), suggesting that many of these genes were the direct or indirect targets of the induced transcription factors. Simulation studies, as well as gene-specific RNA half-life data (15), showed that differential degradation rates of RNA species (11) could not explain the observed class preferences (supplementary text and figs. S7 and S8). Although these patterns were evident across cell types and species, few promoters (mean $8.5 \%$ across classes) and even fewer enhancers (mean $5.1 \%$ across classes) were assigned to the same response class in two or more time courses (Fig. 1F).

We looked further at a literature-curated set of 232 immediate early response (IER) genes (table S5). Although $65 \%$ of the IER genes had at least one promoter that was up-regulated within the first 2 hours in at least two time courses, no consistent pattern of IER expression was obvious between time courses (fig. S9). For example, only 42 promoters were induced early in five or more human time courses (fig. S10A). Even fewer enhancers shared an early response: Only 11 were induced in three or more time courses (fig. S10B), and of these, half neighbored a known IER gene. Thus, the IER pattern is generalizable across different cell states, but the cohort of IER genes are not.

In general, up-regulated enhancers in the rapid short response class were transcribed earlier than their proximal ( $\pm 200 \mathrm{~kb}$ ) promoters (Fig. 2, A and $\mathrm{B}$, and fig. S11). Proximal TF promoters were, in turn, more highly and more rapidly activated than proximal non-TF promoters. To compare the responses over time, we used the "center of mass" (CM) statistic identifying the time point by which $50 \%$ of the expression change in the enhancer or promoter had occurred. Enhancers changed most rapidly, followed by TF promoters, then non-TF promoters (Fig. 2C). The temporal differences were highlighted further when enhancers were compared to their proximal promoters (within $\pm 200 \mathrm{~kb}$ ) (Fig. 2C). For $85.8 \%$ of enhancer-non-TF promoter pairs and $74.6 \%$ of 
enhancer-TF promoter pairs, the $\mathrm{CM}$ occurred earlier for the enhancer $\left(P<1.0 \times 10^{-106}\right.$, Wilcoxon signed rank test). We hypothesized that these results might reflect larger chromatin structures; indeed, enhancer-promoter pairs defined by topological domains (TADs) (16) gave similar results (figs. S11 and S12), and moreover, enhancers (or promoters) within the same TAD were more likely to be in the same response class (Fig. 2D). Similarly, groups of enhancer-promoter pairs (defined either by genomic proximity or TAD boundaries) were more similar in terms of CM shifts than expected by chance (fig. S13, $P<1.0 \times 10^{-14}$, Mann-Whitney U test).

We used ENCODE (13) data to demonstrate that enhancers dynamically expressed in the MCF-7+HRG time course were more likely to be marked with high deoxyribonuclease (DNAse) sensitivity and enriched in H3K27ac and RNA polymerase II (RNAPII) chromatin immunoprecipitation signal in steady-state MCF-7 cells than enhancers that were not active throughout the time course (fig. S14A). Indeed, chromatin interaction analysis with paired-end-tag sequencing (ChIA-PET) data from steady-state MCF-7 cells (17) showed that these dynamic enhancers interacted with promoters to a much larger extent than nonactive enhancers, but the fraction of promoter-interacting enhancers was high regardless of response class (Fig. 2E), suggesting that many dynamically changing enhancers are proximal to their promoter target(s) and primed beforehand in terms of chromatin state. However, chromatin patterns in the unstimulated state were not sufficient to distinguish between temporal enhancer classes (fig. S14B).

Transcription factor binding sites implicated in regulating enhancer and promoter expression were assessed by inferring motif activities (18)-a statistic that describes the ability of a DNA motif to explain observed expression changes across a given set of samples-based on motif occurrence in the regions -300 to +100 base pairs (bp) from the major TSSs of each promoter and $\pm 200 \mathrm{bp}$ from the center of each enhancer, resulting in a derived activity profile across time for each DNA binding motif and time course. Motif sets with high predictive power in enhancers and promoters overlapped significantly (false discovery rate $<0.05$, Fisher's exact test) in 29 out of 33 time courses (Fig. 3A). Many of these highly contributing motifs described binding sites for known lineage-specific regulators in specific time courses, such as FOS in MCF-7 cells stimulated by HRG, GATA6 in cardiomyocyte differentiation, and nuclear factor $\kappa \mathrm{B}(\mathrm{NF}-\kappa \mathrm{B})$ in macrophages. On average, motif activity scores correlated positively across time between enhancers and promoters, with significantly higher correlation for motifs identified as significantly active (supplementary text) in both enhancers and promoters $\left(P<6.9 \times 10^{-8}\right.$, Mann-Whitney test) (Fig. 3B); however, in general, motif activity reached a maximum in enhancers earlier than in promoters $\left(P<1.8 \times 10^{-14}\right.$, Wilcoxon signed rank test; Fig. 3, C and D). Thus, the general observation of enhancer transcription waves preceding those of promoters identified above was supported by motif activity.

In summary, by using a large-scale comparative analysis across many different tissues and time courses, and simultaneously sampling expression at gene promoters and enhancers, we reveal that enhancer transcription is the most common rapid transcription change occurring when cells initiate a state change. Enhancer RNA concentration peaked as early as $15 \mathrm{~min}$ after the transition trigger was applied in some time courses. Although earlier studies of single time courses have reported enhancer activity before gene activation in a small set of enhancer-gene pairs $(8,9,19)$, we can now establish this phenomenon as a general feature of mammalian transcriptional regulation, across a multitude of biological systems. This challenges previous models that suggested that linked enhancers and promoters are coexpressed over

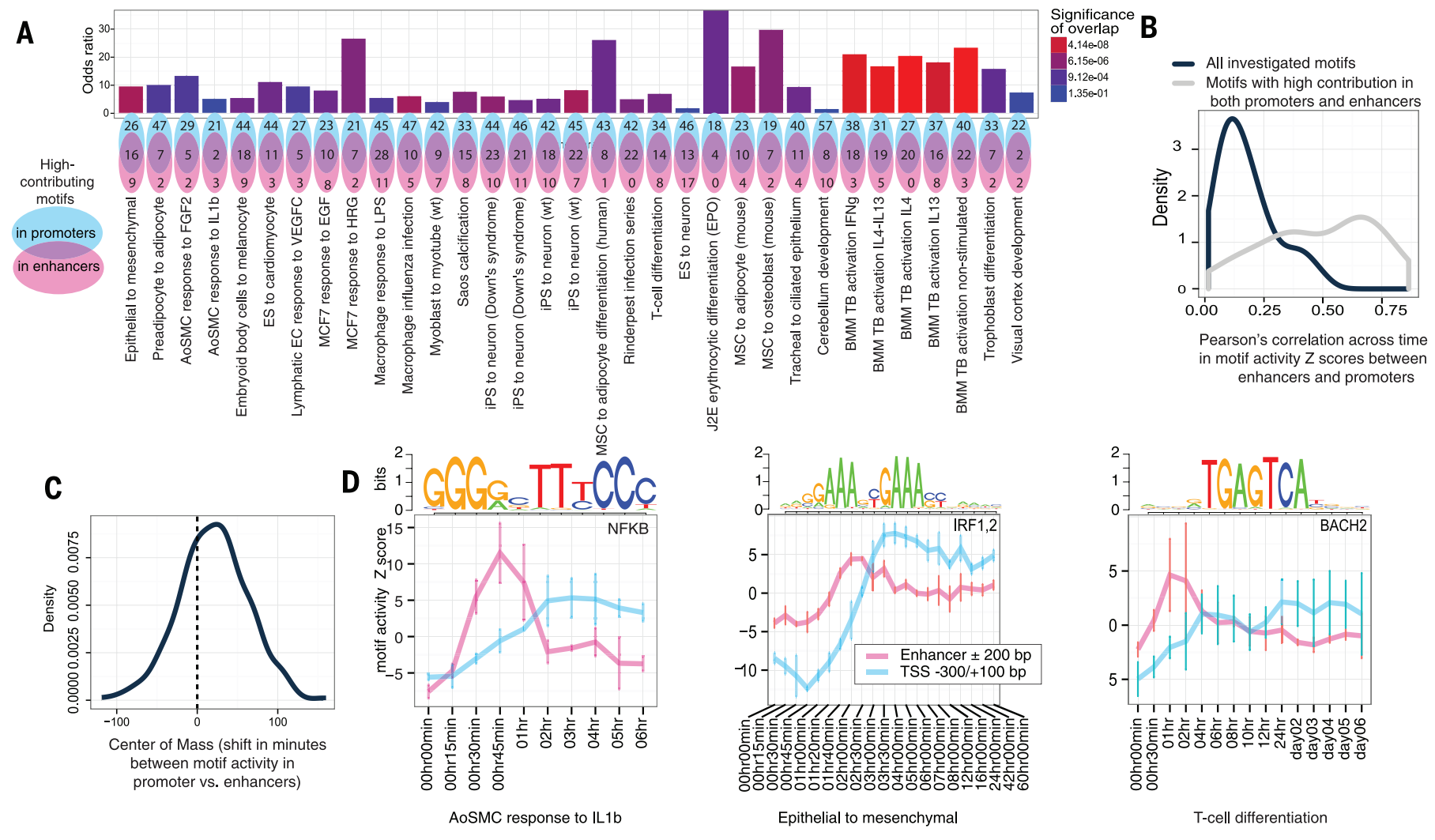

Fig. 3. Motif analysis of linked enhancers and promoters over time. (A) Overlap of motifs classified as significant for driving expression in enhancers and promoters. Top row: bar plot of motif overlap odds ratios, colored by significance. Bottom row: Venn diagrams of motif set overlap. (B) Distributions of average Pearson correlation coefficient between motif activities in enhancers and promoters in all motifs investigated (black) and motifs significantly active in both enhancers and promoters (gray). (C) Distribution of shift (minutes) in motif activity center of mass (see Fig. 2D) in promoters compared to enhancers. (D) Examples of motif activity in enhancers preceding that of promoters. Motif activity is plotted as the average of activity Z scores per time point. Error bars indicate the SD. 
time [e.g., $(8,15,19,20)]$. Indeed, even in the case of late response classes, candidate enhancers appear to be activated in advance of promoters in their vicinity (fig. S11). The rapid burst of eRNA activity at 15 min was frequently followed by a rapid return to baseline (Fig. 1D). In these cases, it may be that once the target promoter has been activated, enhancer activity is no longer required. Other enhancers were rapidly activated and then continuously expressed. These eRNAs may have additional functional roles, such as the recently suggested role in promoting elongation (15).

\section{REFERENCES AND NOTES}

1. M. Levine, C. Cattoglio, R. Tjian, Cell 157, 13-25 (2014)

2. S. Bonn et al., Nat. Genet. 44, 148-156 (2012).

3. A. S. Nord et al., Cell 155, 1521-1531 (2013).

4. A. B. Stergachis et al., Cell 154, 888-903 (2013).

5. T.-K. Kim et al., Nature 465, 182-187 (2010)

6. H. Wu et al., PLOS Genet. 10, e1004610 (2014).

7. M. Rönnerblad et al., Blood 123, e79-e89 (2014).

8. M. U. Kaikkonen et al., Mol. Cell 51, 310-325 (2013).

9. N. Hah, S. Murakami, A. Nagari, C. G. Danko, W. L. Kraus, Genome Res. 23, 1210-1223 (2013).

10. W. Li et al., Nature $498,516-520$ (2013).

11. R. Andersson et al., Nat. Commun. 5, 5336 (2014).

12. R. Andersson et al., Nature 507, 455-461 (2014).

13. ENCODE Project Consortium, Nature 489, 57-74 (2012).

14. FANTOM Consortium and the RIKEN PMI and CLST (DGT) Nature 507, $462-470$ (2014).

15. K. Schaukowitch et al., Mol. Cell 56, $29-42$ (2014).

16. J. R. Dixon et al., Nature 485, 376-380 (2012)

17. G. Li et al., Cell 148, 84-98 (2012).

18. FANTOM Consortium et al., Nat. Genet. $41,553-562$ (2009).

19. C.-L. Hsieh et al., Proc. Natl. Acad. Sci. U.S.A. 111, 7319-7324 (2014).

20. N. E. Ilott et al., Nat. Commun. 5, 3979-3979 (2014).

\section{ACKNOWLEDGMENTS}

For a full list of acknowledgements and contributions, see supplementary text. FANTOM5 was made possible by a Research Grant for RIKEN Omics Science Center from the Ministry of Education, Culture, Sports, Science, and Technology of Japan (MEXT) to Y. Hayashizaki. It was also supported by Research Grants for RIKEN Preventive Medicine and Diagnosis Innovation Program to Y. Hayashizaki and RIKEN Centre for Life Science Technologies, Division of Genomic Technologies (from the MEXT, Japan). Additional funding is listed in the supplementary text. All CAGE data needed to reproduce the study have been deposited at the DNA Data Bank of Japan (DDBJ) under accession numbers DRA000991, DRA002711, DRA002747, and DRA002748. Additional visualizations of the data are available at http://fantom. gsc.riken.jp/5/. The human induced pluripotent stem cell lines that were subjected to cortical neuronal differentiation can be made available after completion of a materials transfer agreement with the Australian Institute for Bioengineering and Nanotechnology of The University of Queensland.

\section{SUPPLEMENTARY MATERIALS}

www.sciencemag.org/content/347/6225/1010/suppl/DC1 Materials and Methods

Supplementary Text

Figs. S1 to S14

Tables S1 to S5

Auxiliary data tables S1 to S3

References (21-32)

31 July 2014; accepted 26 January 2015

Published online 12 February 2015

10.1126/science. 1259418

\title{
Evolutionary resurrection of flagellar motility via rewiring of the nitrogen regulation system
}

\author{
Tiffany B. Taylor, ${ }^{1 *}$ Geraldine Mulley, ${ }^{\text {1* }}$ Alexander H. Dills, ${ }^{2}$ Abdullah S. Alsohim, ${ }^{1,3}$ \\ Liam J. McGuffin, ${ }^{1}$ David J. Studholme, ${ }^{4}$ Mark W. Silby, ${ }^{2}$ Michael A. Brockhurst, ${ }^{5}$ \\ Louise J. Johnson, ${ }^{1} \dagger$ Robert W. Jackson ${ }^{1,6}$
}

A central process in evolution is the recruitment of genes to regulatory networks. We engineered immotile strains of the bacterium Pseudomonas fluorescens that lack flagella due to deletion of the regulatory gene fleQ. Under strong selection for motility, these bacteria consistently regained flagella within 96 hours via a two-step evolutionary pathway. Step 1 mutations increase intracellular levels of phosphorylated NtrC, a distant homolog of FleQ, which begins to commandeer control of the fleQ regulon at the cost of disrupting nitrogen uptake and assimilation. Step 2 is a switch-of-function mutation that redirects $\mathrm{NtrC}$ away from nitrogen uptake and toward its novel function as a flagellar regulator. Our results demonstrate that natural selection can rapidly rewire regulatory networks in very few, repeatable mutational steps.

A long-standing evolutionary question concerns how the duplication and recruitment of genes to regulatory networks facilitate their expansion (1) and how networks gain mutational robustness and evolvability (2). Bacteria respond to diverse environments using a vast range of specialized regulatory pathways, predominantly two-component systems (3), which are the result of adaptive radiations within gene families. Due to past cycles of gene duplication, divergence, and horizontal genetic transfer, there is often extensive homology between the components of different pathways (4), raising the possibility of cross-talk or redundancy between pathways (5). Here we monitor the recovery of microbial populations from a catastrophic gene deletion: Bacteria engineered to lack a particular function are exposed to environments that impose strong selection to re-evolve it, sometimes by recruitment of genes to regulatory networks (6-9).

In the plant-associated soil bacterium Pseudomonas fluorescens, the master regulator of flagellar synthesis is FleQ (also called AdnA), a $\sigma^{54}$-dependent enhancer binding protein (EBP) that activates transcription of genes required for flagellum biosynthesis $(10,11)$. The starting $P$. fluorescens strain is AR2; this strain lacks flagella due to deletion of fleQ and is unable to move by spreading motility due to mutation of viscosin synthase (viscB), resulting in a distinctive, pointlike colony mor-

${ }^{1}$ School of Biological Sciences, University of Reading, Whiteknights, Reading RG6 6AJ, UK. '2Department of Biology, University of Massachusetts Dartmouth, 285 Old Westport Road, North Dartmouth, MA 02747, USA. ${ }^{3}$ Department of Plant Production and Protection, Qassim University, Qassim, P.0. Box 6622. Saudi Arabia. ${ }^{4}$ College of Life and

Environmental Sciences, University of Exeter, Stocker Road, Exeter EX4 4QD, UK. ${ }^{5}$ Department of Biology, University of York. Wentworth Way, York Y010 5DD, UK. ${ }^{6}$ The University of Akureyri, Borgir vid Nordurslod, IS-600 Akureyri, Iceland.

*These authors contributed equally to this work. †Corresponding author. E-mail: I.j.johnson@reading.ac.uk phology on spreading motility medium (SMM) (12) (Fig. 1A). We grew replicate populations of AR2 on SMM (see supplementary materials and methods); when local nutrients became depleted, starvation imposed strong selection to re-evolve motility. To demonstrate that this finding was not strain-specific, we replicated these experiments in a different strain of $P$. fluorescens, Pf0-2x. This strain is a $\triangle$ fle $Q$ variant of Pf0-1, already viscosindeficient, and thus unable to move by spreading or swimming motility (10).

After 96 hours of incubation of AR2 and Pf0-2x at room temperature on SMM, two breakout mutations were visible, conferring first slow (AR2S and PfO-2xS) and then fast (AR2F and PfO-2xF) spreading over the agar surface (Fig. 1A). The AR2F strain produces flagella, but we could not detect flagella in electron microscopy samples for AR2S (Fig. 1B). Genome resequencing revealed a single-nucleotide point mutation in $n t r B$ in strain AR2S, causing an amino acid substitution within the PAS domain of the histidine kinase sensor NtrB $\left[\mathrm{Thr}^{97} \rightarrow \mathrm{Pro}^{97}\right.$ (T97P)] (13). The fastspreading strain AR2F had acquired an additional point mutation in the $\sigma^{54}$-dependent EBP gene $n t r C$, which alters an amino acid (R442C) within the DNA binding domain (Table 1 and table S2).

NtrB and NtrC make up a two-component system: Under nitrogen limitation, NtrB phosphorylates NtrC, which activates transcription of genes required for nitrogen uptake and metabolism. To determine how mutations in this separate regulatory pathway restored motility in the absence of FleQ, we performed microarray and quantitative reverse transcription polymerase chain reaction analyses of the ancestral and evolved strains (fig. S1 and table S1). The expression of genes required for flagellum biosynthesis and chemotaxis was abolished in AR2 compared with wildtype (WT) SBW25 (Fig. 2A). The ntrB mutation in AR2S partially restores the expression of 


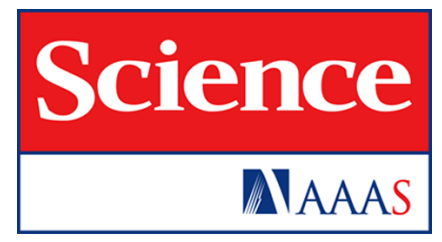

Transcribed enhancers lead waves of coordinated transcription in transitioning mammalian cells

Erik Arner, Carsten O. Daub, Kristoffer Vitting-Seerup, Robin Andersson, Berit Lilje, Finn Drabløs, Andreas Lennartsson, Michelle Rönnerblad, Olga Hrydziuszko, Morana Vitezic, Tom C. Freeman, Ahmad M. N. Alhendi, Peter Arner, Richard Axton, J. Kenneth Baillie, Anthony Beckhouse, Beatrice Bodega, James Briggs, Frank Brombacher, Margaret Davis, Michael Detmar, Anna Ehrlund, Mitsuhiro Endoh, Afsaneh Eslami, Michela Fagiolini, Lynsey Fairbairn, Geoffrey J. Faulkner, Carmelo Ferrai, Malcolm E. Fisher, Lesley Forrester, Daniel Goldowitz, Reto Guler, Thomas Ha, Mitsuko Hara, Meenhard Herlyn, Tomokatsu Ikawa, Chieko Kai, Hiroshi Kawamoto, Levon M. Khachigian, S. Peter Klinken, Soichi Kojima, Haruhiko Koseki, Sarah Klein, Niklas Mejhert, Ken Miyaguchi, Yosuke Mizuno, Mitsuru Morimoto, Kelly J. Morris, Christine Mummery, Yutaka Nakachi, Soichi Ogishima, Mariko Okada-Hatakeyama, Yasushi Okazaki, Valerio Orlando, Dmitry Ovchinnikov, Robert Passier, Margaret Patrikakis, Ana Pombo, Xian-Yang Qin, Sugata Roy, Hiroki Sato, Suzana Savvi, Alka Saxena, Anita Schwegmann, Daisuke Sugiyama, Rolf Swoboda, Hiroshi Tanaka, Andru Tomoiu, Louise N. Winteringham, Ernst Wolvetang, Chiyo Yanagi-Mizuochi, Misako Yoneda, Susan Zabierowski, Peter Zhang, Imad Abugessaisa, Nicolas Bertin, Alexander D. Diehl, Shiro Fukuda, Masaaki Furuno, Jayson Harshbarger, Akira Hasegawa, Fumi Hori, Sachi Ishikawa-Kato, Yuri Ishizu, Masayoshi Itoh, Tsugumi Kawashima, Miki Kojima, Naoto Kondo, Marina Lizio, Terrence F. Meehan, Christopher J. Mungall, Mitsuyoshi Murata, Hiromi Nishiyori-Sueki, Serkan Sahin, Sayaka Nagao-Sato, Jessica Severin, Michiel J. L. de Hoon, Jun Kawai, Takeya Kasukawa, Timo Lassmann, Harukazu Suzuki, Hideya Kawaji, Kim M. Summers, Christine Wells, FANTOM Consortium, David A. Hume, Alistair R. R. Forrest, Albin Sandelin, Piero Carninci and Yoshihide Hayashizaki (February 12, 2015) Science 347 (6225), 1010-1014. [doi: 10.1126/science.1259418] originally published online February 12, 2015

Science (print ISSN 0036-8075; online ISSN 1095-9203) is published weekly, except the last week in December, by the American Association for the Advancement of Science, 1200 New York Avenue NW, Washington, DC 20005. Copyright 2016 by the American Association for the Advancement of Science; all rights reserved. The title Science is a registered trademark of AAAS. 


\section{Editor's Summary}

\section{Uncaging promoter and enhancer dynamics}

In order to understand cellular differentiation, it is important to understand the timing of the regulation of gene expression. Arner et al. used cap analysis of gene expression (CAGE) to analyze gene enhancer and promoter activities in a number of human and mouse cell types. The RNA of enhancers was transcribed first, followed by that of transcription factors, and finally by genes that are not transcription factors.

Science, this issue p. 1010

This copy is for your personal, non-commercial use only.

Article Tools Visit the online version of this article to access the personalization and article tools:

http://science.sciencemag.org/content/347/6225/1010

Permissions Obtain information about reproducing this article:

http://www.sciencemag.org/about/permissions.dtl

Science (print ISSN 0036-8075; online ISSN 1095-9203) is published weekly, except the last week in December, by the American Association for the Advancement of Science, 1200 New York Avenue NW, Washington, DC 20005. Copyright 2016 by the American Association for the Advancement of Science; all rights reserved. The title Science is a registered trademark of AAAS. 